EPJ Web of Conferences 97,00010 (2015)

DOI: $10.1051 /$ epjconf/20159700010

(C) Owned by the authors, published by EDP Sciences, 2015

\title{
Resonances at COSY — the dibaryon issue
}

\author{
H. Clement ${ }^{1,2, a}$, M. Bashkanov ${ }^{1,2}$, and T. Skorodko ${ }^{3}$ \\ ${ }^{1}$ Physikalisches Institut der Universität Tübingen, Auf der Morgenstelle 14, D-72076 Tübingen, Germany \\ ${ }^{2}$ Kepler Center for Astro and Particle Physics, University of Tübingen, Auf der Morgenstelle 14, D-72076 \\ Tübingen, Germany \\ ${ }^{3}$ Department of Physics, Tomsk State University, 36 Lenina Avenue, Tomsk 634050, Russia
}

\begin{abstract}
The cooler synchrotron COSY at the research center Jülich has been dedicated to the investigation of nucleon-nucleon collisions. Together with appropriate detector setups it has been particularly well suited for the search of resonances in the baryonbaryon system. Hence it is of no surprise that the observation of the first non-trivial dibaryon resonance has been established at this machine. A review on this issue is given - including the quest for dibaryons in nuclear matter.
\end{abstract}

\section{Introduction}

After twenty years of successful operation the hadron physics program has been finished by the end of 2014 at the cooler synchrotron COSY installed at the research center Jülich. The primary goal was the investigation of the nucleon-nucleon interaction in the energy range between $\pi$ and $\phi$ production thresholds. This allowed detailed studies of the production and decay of meson and baryon resonances in this energy range. Long-term programs, which have been carried out, include the elastic protonproton scattering with polarized beam and target at the EDDA set-up, the strangeness production in proton-proton and proton-neutron collisions at ANKE and TOF as well as the $\eta$ decay studies at the WASA detector, which had been moved from CELSIUS to COSY in 2005.

The WASA detector $[1,2]$ with a nearly full solid angle coverage and its possibility to measure the four-momenta of both neutral and charged particles has been ideally suited for the study of reactions, where many particles are emitted - such as multi-pion production. In combination with COSY delivering a cooled beam with high momentum resolution this setup was uniquely adapted to search for narrow resonances in the nucleon-nucleon collision process. In the following a review is given on the finally successful search for dibaryon resonances.

\section{Dibaryons}

\subsection{The early days}

The question, whether there are more eigenstates in the system of two baryons, has been around in principle since the discovery of the deuteron in 1932. Since it was clear very soon that the loosely

\footnotetext{
ae-mail: heinz.clement@uni-tuebingen.de
} 
bound deuteron would not have any excited bound states, the search for resonances focused soon on nucleon-nucleon collision energies close to the pion-production threshold and above. Reports on such measurements date back to the fifties, when suitable particle accelerators became available.

The quest for dibaryon states was reinforced, when it got apparent that baryons and mesons contain substructures, the quarks, and QCD does not prohibit colorless multiples of three quarks, in particular does not forbid quarks in a colorless six-pack. In fact, shortly after Gell-Mann's famous publication [3] of the quark model in 1964 Dyson and Xuong [4] demonstrated that SU(6) symmetry breaking provides six non-strange dibaryon sates. The first three they identified as the deuteron groundstate, the virtual ${ }^{1} S_{0}$ isovector state - known at that time already from the final-state interaction in low-energy nucleon-nucleon scattering - and an $I\left(J^{p}\right)=1\left(2^{+}\right)$state right at the $N \Delta$ threshold, for which first experimental indications had been available already at that time - see discussion in Ref. [4].

However, it was only in the eighties and nineties, when high-quality data for $p p$ and $\pi d$ elastic scattering as well as for the $\pi d \rightarrow p p$ reaction in the relevant energy regime became available and allowed a sophisticated partial-wave analysis. As a result the SAID data analysis group could demonstrate that the ${ }^{1} D_{2}$ partial wave in the $p p$ system is, indeed, resonating near the $N \Delta$ threshold producing a pronounced looping in the Argand diagram [5-7]. Usually this is a clear indication of a genuine $s$-channel resonance. However, the fact that this happens just close to the $N \Delta$ threshold exhibiting a width comparable to that of the $\Delta$ resonance, makes it difficult to exclude that this looping is merely a reflection of the conventional $\Delta$ excitation in the presence of a nucleon with both being at rest relative to each other due to the threshold condition. For the discussions, whether this phenomenon constitutes a genuine $s$-channel resonance or just a threshold cusp or virtual state see, e.g., Refs. [8-12].

\subsection{The dibaryon rush era}

A real dibaryon rush started, when Jaffe 1977 [13] predicted the so-called H-dibaryon, a bound $\Lambda \Lambda$ system containing two strange quarks. This initiated a flood of further predictions on a multitude of states in all kind of baryon-baryon systems, which in turn initiated world-wide experimental searches for bound and un bound dibaryon states. As a consequence a huge number of claims have been made. However, finally not a single one survived a critical inspection.

A major reason for this striking failure was certainly the insufficient quality of experimental data obtained by use of inadequate instrumental equipment. Other reasons may concern the wrong choice of reaction and/or energy region, where searches have been conducted. For a critical, but also amusing review of this epoch see, e.g. K. K. Seth [12].

\subsection{Exclusive and kinematically complete measurements}

A conclusion drawn from the experiences made in the dibaryon rush era is that any meaningful dibaryon search should be undertaken with a dedicated experimental equipment, which is suitable to provide exclusive and kinematically complete measurements of high statistics and high fourmomentum resolution. In the nineties two programs were set up at CELSIUS and COSY, which allowed the search for dibaryon resonances with dedicated equipment within systematic studies of elastic scattering and two-pion production, respectively.

The EDDA experiment at COSY provided the ideal requirements for measuring the elastic protonproton scattering over nearly the full angular range both with polarized beam and target. In energy scan runs the energy range between $\pi$ and $\phi$ thresholds could be scanned with sub-MeV resolution in high-statistics runs, which would have been able to reveal even very narrow, weakly excited dibaryon 
resonances. Though no indications for any narrow resonances in the proton-proton system have been found, this EDDA data set [14] is of indispensable value, since it constitutes the backbone of highquality proton-proton scattering data in the SAID data base [15] fixing the empirical partial-wave amplitudes with unprecedented precision. Unfortunately the EDDA experiment was not continued to examine also the proton-neutron scattering with similar precision - historically possibly a big mistake, as we will see below.

The WASA experiment, until 2005 at CELSIUS and thereafter at COSY, provided the ideal requirements for measuring multi-pion production in unprecedented quality. Though the $\pi N$ system was known to provide a wealth of baryon resonances and also first meaningful theoretical calculations predicted the $\Delta \Delta$ system to play a dominant role in the two-pion production process, the data base was still scarce at that time. A reason was that afore no detector was available on a nucleonnucleon machine, which was able to cover the full phase space of complex reactions.

Since $p p$ collisions are experimentally much easier to conduct than $p n$ collisions, the WASA collaboration started to systematically measure all $p p$-induced, i.e. isovector two-pion production channels from threshold up to $\sqrt{s}=2.5 \mathrm{GeV}$ [16-24] complemented by a corresponding program with polarized beam at COSY-TOF $[25,26]$. As a result of these systematic studies it was found that isovector induced two-pion production can be quantitatively well understood by the conventional process of $t$-channel meson exchange leading to the excitation of Roper resonance close to threshold followed by the excitation of the $\Delta \Delta$ system at higher energies. To some extent also the $\Delta(1600)$ excitation is seen to play some role. But no hint for an exotic resonance production was observed.

\subsection{Towards the first non-trivial dibaryon}

The situation changed drastically, when $p n$-induced two-pion production was looked at, though the pathway towards a dibaryon resonance was still far from being straight forward. In a first step we looked on the reaction $p d \rightarrow{ }^{3} \mathrm{He} \pi^{0} \pi^{0}$ and $p d \rightarrow{ }^{3} \mathrm{He} \pi^{+} \pi^{-}$, for which data were available from runs dedicated to the $\eta$ production at threshold. This so-called double-pionic fusion reaction to ${ }^{3} \mathrm{He}$ was known for its unsolved problem, the so-called ABC effect, which denotes an unusual low-mass enhancement in the invariant mass spectrum of the two produced pions. This phenomenon, which was observed also in the fusion reactions to $d$ and ${ }^{4} \mathrm{He}$ - if the produced pion pair was isoscalar - did not find a satisfactory theoretical explanation so far. Hence it was called just after the initials of the authors Abashian, Booth and Crowe, who were the first to reported about this effect [27].

Since previous measurements either were low-statistics bubble-chamber or inclusive single-arm measurements conducted at a few scattering angles, we decided to reexamine the ABC effect at WASA with exclusive and kinematically complete measurements. As a result we found [28] that there is, indeed, a highly visible low-mass enhancement and we came to the conclusion that it might be a sign of a quasi-bound $\Delta \Delta$ system. However, if this denotes a resonance phenomenon in the system of two baryons, then it would need to show up also as a resonance structure in the total cross section of the basic double-pionic fusion, which is the one to the deuteron.

For this endeavor the experimental technique had to be changed, since free neutrons are not available - neither as beam or as target particles. Hence we utilized the quasi-free process with deuterons being the source for quasi-free neutrons - a method established before in many other experiments, see e.g. Refs. [29, 30]. This process has the additional advantage that the Fermi motion of the neutron within the deuteron provides a range of collision energies with a single beam energy setting. That way the energy dependence of a reaction can be conveniently scanned, which is particularly well suited for the search for narrow resonances. However, a precondition for a successful use of the quasi-free process is that the four-momenta of all ejectiles are determined experimentally, which necessitates exclusive and kinematically complete measurements. 


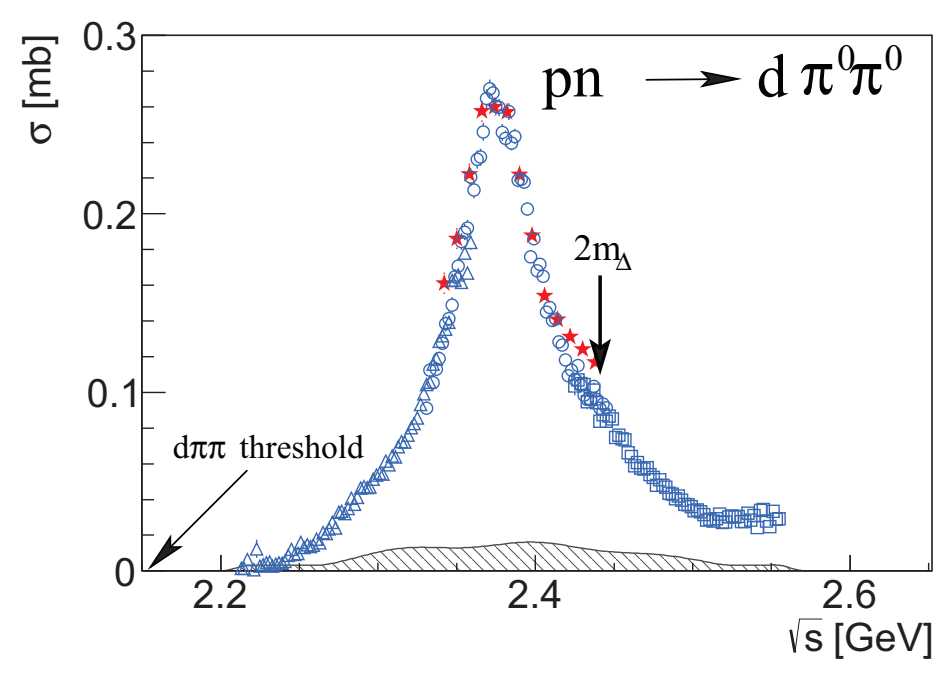

Figure 1. Total cross section of the golden reaction channel $p n \rightarrow d \pi^{0} \pi^{0}$ exhibiting the pronounced resonance effect of $d^{*}(2380)$. The blue open symbols show the data of Ref. [32] properly normalized in absolute scale to the data of Ref. [33] plotted by red stars. The black shaded area gives an estimate of systematic uncertainties.

First such measurements were conducted still with WASA at CELSIUS. Though the statistics was still quite limited, a clear correlation of the $\mathrm{ABC}$ effect with a peak-like structure in the total cross section could be revealed in the $p n \rightarrow d \pi^{0} \pi^{0}$ reaction, which was measured via the quasi-free process $p d \rightarrow d \pi^{0} \pi^{0}+p_{\text {spectator }}$ [31]. As it turned out later, this was the golden channel for the dibaryon issue, since it posesses a very low background from conventional processes. Experimentally it was only accessible with instruments like WASA being able to detect both charged and uncharged particles over essentially the full solid angle. Hence it is not of a surprise that there are no data for this channel from previous experiments.

Follow-up measurements of this reaction - now with WASA at COSY providing two orders of magnitude better statistics - revealed a pronounced Lorentzian structure in the total cross section corresponding to a resonance at $2.37 \mathrm{GeV}$ having a width of only $70 \mathrm{MeV}$ [32], see Fig. 1. Although the resonance structure is about $90 \mathrm{MeV}$ below the mass of two $\Delta$ states and also its width is more than three times narrower than that of a conventional $t$-channel $\Delta \Delta$ excitation, the Dalitz plot of this reaction clearly shows that this resonance structure decays predominantly into a $\Delta \Delta$ system. In addition, the high-statistic angular distributions provided spin-parity of this structure to be $3^{+}$. Together with the fact that the $p n \rightarrow d \pi^{0} \pi^{0}$ reaction is purely isoscalar, we get the quantum numbers $I\left(J^{P}\right)=0\left(3^{+}\right)$. This has been confirmed by subsequent measurements of all three fusion reactions $p n \rightarrow d \pi^{0} \pi^{0}$, $p n \rightarrow d \pi^{+} \pi^{-}$and $p p \rightarrow d \pi^{+} \pi^{0}$ and their isospin decomposition [33].

Recently also the partially isoscalar non-fusion channels $p n \rightarrow p p \pi^{0} \pi^{-}$[34], $p n \rightarrow p n \pi^{0} \pi^{0}$ [35], $p n \rightarrow p n \pi^{+} \pi^{-}$[36] have been investigated. For the latter also HADES is expected to contribute with new measurements. As as result all two-pion production channels are consistent with the hypothesis of an $I\left(J^{P}\right)=0\left(3^{+}\right)$dibaryon resonance at $2.37 \mathrm{GeV}$ with a width of $70 \mathrm{MeV}$. The observed decay branchings into the diverse two-pion channels are consistent with isospin coupling and explicit theoretical calculations [37-39]. 


\section{$2.5 d^{*}(2380)$ - a genuine dibaryon resonance}

In order to prove that the resonance structure observed in two-pion production indeed constitutes a true resonance, i.e. a $s$-channel resonance, it has to be sensed also in the entrance channel, i.e. in $n p$ scattering. There it has to be shown that it produces a pole in the partial waves corresponding to $I\left(J^{P}\right)=0\left(3^{+}\right)$, i.e. in the coupled partial-wave system ${ }^{3} D_{3}-{ }^{3} G_{3}$.

From the knowledge of the resonance contribution to the two-pion channels the expected resonance contribution to elastic $n p$ scattering can be estimated [37] to be in the order of about $170 \mu \mathrm{b}$, which has to be compared to a total $n p$ cross section of nearly $40 \mathrm{mb}$. The only observable, which has the potential to sense such a small contribution, is the analyzing power, since it is composed of only interference terms in the partial waves and hence sensitive to small contributions in partial waves. Due to the spin of the resonance its angular contribution in the analyzing power has to be according to the associated Legendre polynomial $P_{3}^{1}$ and hence has to be largest at $90^{\circ}$, where the differential cross section is lowest.

Following these considerations the analyzing power was measured over the energy region of interest and over the full angular range with WASA at COSY. The experiment was carried out again in the quasi-free mode and in inverse kinematics by use of a polarized deuteron beam hitting the hydrogen pellet target. The subsequent partial-wave analysis of the data by the SAID group, indeed, revealed a pole in the coupled ${ }^{3} D_{3}-{ }^{3} G_{3}$ coupled waves at $(2380 \pm 10)-i(40 \pm 5) \mathrm{MeV}-$ fully consistent with the findings in the two-pion production reactions [40-42].

This result establishes the resonance structure observed in two-pion production as a true $s$-channel resonance in the proton-neutron system. Since it is of isoscalar character we chose $d^{*}(2380)$ as its denotation in analogy to the notation for isoscalar excitations of the nucleon. Also, the notation $d^{*}$ was used already by Goldman et.al. [43] when predicting the so-called "inevitable dibaryon" with identical quantum numbers.

\subsection{Former occasions for a discovery}

Having found now $d^{*}(2380)$ we may ask the question, whether already previous experiments could have had the chance to discover it. As already mentioned above, the golden channel $p n \rightarrow d \pi^{0} \pi^{0}$ was not accessible instrumentally before. But what about other channels?

In the $p n \rightarrow d \pi^{+} \pi^{-}$reaction the resonance effect is no longer as pronounced, but still could have been observed in principle by previous bubble chamber experiments at DESY [44] and JINR [45] in case of much improved statistics as well as neutron beam energy resolution and energy scan, respectively.

Previous $n p$ scattering experiments at LAMPF and Saclay were limited by the maximum beam energy available at these accelerator facilities, which were below the energy region of interest here. The EDDA experiment at COSY would have had a great chance of its discovery, if its $p p$ scattering program would have been complimented by a corresponding $n p$ scattering program - as intended initially.

Actually Japanese groups had been quite close already as early as 1977 considering measurements of the proton polarization in deuteron photodisintegration $\gamma d \rightarrow \vec{p} n$ [46]. They observed a strong increase of the polarization at energies beyond $\sqrt{s}=2.2 \mathrm{GeV}$, reaching a maximum at $2.38 \mathrm{GeV}$ and decreasing thereafter. From the fact that the effect appeared to be largest at $90^{\circ}$ they concluded that possibly an $I\left(J^{P}\right)=0\left(3^{+}\right)$deeply bound $\Delta \Delta$ system could be the reason - making also a possible connection to $\mathrm{ABC}$ effect and two-pion production channels. Only, their data favored a broad resonance of about $160 \mathrm{MeV}$ width. Follow-up measurements and their analysis showed that the data and their analysis are best described by the assumption of several broad resonances of different spin-parity with 
widths larger than $200 \mathrm{MeV}$ [47]. From the large widths it looks that these findings correspond to $t$-channel $\Delta \Delta$ excitations rather than the narrow $d^{*}(2380)$ dibaryon resonance. However, we note that recent Jlab measurements of the proton polarization at $90^{\circ}$ [48] confirm a maximum polarization of -1 at $\sqrt{s}=2.38 \mathrm{GeV}$ with a sharp decrease at higher energies. This indicates that on top of polarization effects due to conventional $t$-channel $\Delta \Delta$ excitations there might be, indeed, a visible effect from $d^{*}(2380)$. If true, it would open the door to measurements of the electromagnetic excitation of $d^{*}(2380)$ in such reactions providing access to the formfactor and thus to the size of this object.

\section{$2.7 d^{*}(2380)$ in nuclear matter}

If dibaryons exist and if they even survive in a nuclear surrounding, then they should have an impact on the nuclear equation of state [49-51], which is of relevance not only for heavy-ion collisions but also for very compact stellar objects like neutron stars.

As already expected from the ABC effect observed previously in the double-pionic fusion reactions to the He isotopes, the exclusive and kinematically complete measurements of these fusion reactions conducted with WASA at COSY could demonstrate $[52,53]$ that indeed $d^{*}(2380)$ is present also in these reactions albeit with an increased width due to Fermi motion in these nuclei.

To investigate this question for still heavier nuclei by exclusive double-pionic fusion reactions gets experimentally very difficult, since the kinetic energies of the fusion products get below the detection thresholds of traditional experimental setups and would afford new, very sophisticated instruments.

An indication for the existence of $d^{*}(2380)$ in nuclear matter may come from heavy-ion collisions in connection with the so-called DLS puzzle. In relativistic heavy-ion collisions an unusual enhancement has been observed in the spectrum of emitted $e^{+} e^{-}$-pairs with invariant masses between $\pi$ and $\omega$ mass. First interpreted as a possible signal from the quark-gluon plasma it was soon realized that this enhancement persists also at low collision energies. Dedicated measurements of diplepton production in nucleon-nucleon collisions by DLS [54] and HADES [55] could trace back this phenomenon to a non-understood enhancement in $p n$ collisions at energies below $\sqrt{s} \approx 3 \mathrm{GeV}$. In a recent publication [56] we could demonstrate that two-pion production via $t$-channel $\Delta \Delta$ and $d^{*}$ (2380) excitation, where the produced $\rho$-channel pion pair transforms into a lepton pair, can account quantitatively for this enhancement and thus solve the DLS-puzzle. Since this enhancement is observed also in heavy-ion collisions, this means in turn that obviously also $d^{*}(2380)$ has been produced in those collisions.

\section{$2.8 d^{*}(2380)$ in theory}

As already mentioned at the beginning of Sect. 2 the very first theoretical paper on dibaryons in connection with quarks - or more precise with SU(6) symmetry breaking, the one by Dyson and Xuong in 1964 [4], predicted properly the now discovered state $d^{*}(2380)$ - at a mass remarkably close to the experimentally observed one. Subsequent, partially very complex theoretical investigations, turned out to be much less successful. In particular they predicted often a multitude of states, which were never observed.

In connection with the observation of large polarization effects in deuteron photodisintegration (see Sect. 2.6) also Kamae and Fujita [46] predicted 1977 the mass of the now observed state properly based on a non-relativistic one-boson exchange potential model for two $\Delta$ isobars.

Goldman et al. [43] pointed out 1989 that due to the unique symmetries of such a state any model based on confinement and effective one-gluon exchange must predict the existence of this state calling it the "inevitable" dibaryon. However, initially they predicted an enormous binding energy as large as $350 \mathrm{MeV}$ for such a state. Only recently - after the experimental observation - the calculations of this group approached the experimental value [57]. 
Meanwhile, there are also other QCD-based calculations, which find this state at about the right mass $[58,59]$. Recently also a relativistic Faddeev-type calculation based on hadronic interactions have been reported to see this state correctly at the experimental mass [60].

Common to all but one [58] theoretical investigations so far is that they overestimate the width of $d^{*}(2380)$, if they make predictions for the width at all. This may possibly be related to exotic contributions to this state - like, e.g., hidden color aspects as discussed in Refs. [58, 61].

\subsection{Conclusions and outlook}

After having established experimentally the first non-trivial dibaryon state all four states predicted by Dyson and Xuong to be coupled to the $N N$ system have been observed. The remaining two states of the predicted sextet are $N N$-decoupled having I $=2$ and 3, respectively, but may be searched for in $N N$-initiated multi-pion production. The latter one with mirrored quantum numbers relative to $d^{*}$ appears to be particularly interesting [61]. Though the hadron physics program has finished at COSY, there is still a wealth of WASA data available, which are suited to search also for these states.

\section{Acknowledgments}

We are indebted to the WASA collaboration and the COSY staff, without who this work would not have been possible and we are grateful to many people for valuable discussions, in particular to those, who were very sceptical about the dibaryon issue and thus forced us to go through all the cumbersome tests of the dibaryon hypothesis. This work has been supported by BMBF, COSY-FFE and DFG(CL 214/3-1).

\section{References}

[1] Chr. Bargholtz et al., Nucl. Inst. Meth. A 594, 339 (2008).

[2] H. H. Adam et al., arxiv: nucl-ex/0411038.

[3] M. Gell-Mann, Phys. Lett. B 8, 214 (1964).

[4] F. J. Dyson and N.-H. Xuong, Phys. Rev. Lett. 13, 815 (1964) and references therein.

[5] R. A. Arndt, I. Strakovsky, R. L. Workman and D. V. Bugg, Phys. Rev. C 48, 1926 (1993).

[6] R. A. Arndt, I. Strakovsky and R. L. Workman, Phys. Rev. C 50, 1796 (1994).

[7] C. H. Oh, R. A. Arndt, I. Strakovsky and R. L. Workman, Phys. Rev. C 56, 635 (1997).

[8] R. L. Shypit et al., Phys. Rev. Lett. 60, 901 (1988); Phys. Rev. C 40, 2203 (1989).

[9] V. V. Anisovich, A. V. Sarantsev and D. V. Bugg, Nucl. Phys. A 537, 501 (1992).

[10] M. G. Ryskin and I. I. Strakovsky, Phys. Rev. Lett. 61, 2384 (1988).

[11] N. Hoshizaki, Phys. Rev. C 45, R1414 (1992); Prog. Theor. Phys. 89, 245, ibid. 251, 563, 569 (1993).

[12] K. K. Seth, Proc. BARYON-BARYON INTERACTION AND DIBARYONIC SYSTEMS, Bad Honnef 1988, 41 (1988)

[13] R. L. Jaffe, Phys. Rev. Lett. 38, 195 and 617(E) (1977).

[14] M. Altmeier et al., Eur. Phys. J. A 23, 351 (2005) and references therein.

[15] SAID database, http://gwdac.phys.gwu.edu/; R.A. Arndt et al., Phys. Rev. C 76,025209 (2007).

[16] W. Brodowski et al., Phys. Rev. Lett. 88, 192301 (2002).

[17] J. Johanson et al., Nucl. Phys. A 712, 75 (2002). Phys. Rev. Lett. 88, 192301 (2002).

[18] J. Pätzold et al., Phys. Rev. C 67, 052202 (2003). 
[19] T. Skorodko et al., Eur. Phys. J. A 35, 317 (2008).

[20] T. Skorodko et al., Phys. Lett. B 679, 30 (2009).

[21] F. Kren et al., Phys. Lett. B 684, 110 (2010) and B 702, 312 (2011); arXiv:0910.0995[nucl-ex]

[22] T. Skorodko et al., Phys. Lett. B 695, 115 (2011).

[23] T. Skorodko et al., Eur. Phys. J. A 47, 108 (2011).

[24] P. Adlarson et al., Phys. Lett. B 706, 256 (2011).

[25] S. Abd El-Bary et al., Eur. Phys. J. A 37, 267 (2008).

[26] S. Abd El-Samad et al., Eur. Phys. J. A 42, 159 (2009).

[27] A. Abashian, N. E. Booth and K. M. Crowe, Phys. Rev. Lett. 5, 258 [1960]; 7, 35 (1961); Phys. Rev. 132, 2296 (1963).

[28] M. Bashkanov et al., Phys. Lett. B 637, 223 (2006).

[29] M. Abdel-Bary et al., Eur. Phys. J. A 29, 353 (2006).

[30] R. Bilger et al., Nucl. Instr. Meth.A 457, 64 (2001).

[31] M. Bashkanov et al., Phys. Rev. Lett. 102, 052301 (2009).

[32] P. Adlarson et al., Phys. Rev. Lett. 106, 242302 (2011).

[33] P. Adlarson et al., Phys. Lett. B 721, 229 (2013).

[34] P. Adlarson et al., Phys. Rev. C 88, 055208 (2013).

[35] P. Adlarson et al., Phys. Lett. B 743, 325 (2015); arxiv:1409.2659 [nucl-ex].

[36] H. Clement, M. Bashkanov and T. Skorodko, Phys. Scr., in press.

[37] M. Bashkanov, H. Clement and T. Skorodko, arxiv:1502.07156 [nucl-ex].

[38] G. Fäldt and C. Wilkin, Phys. Lett. B 701, 619 (2001).

[39] M.Albaladejo and E. Oset, Phys. Rev. C 88, 014006 (2013).

[40] P. Adlarson et al., Phys. Rev. Lett. 112, 202301 (2014).

[41] P. Adlarson et al., Phys. Rev. C 90, 035204 (2014).

[42] R. Workman, EPJ Web Conf. 81, 02023 (2014).

[43] T. Goldman et al., Phys. Rev. C 39, 1889 (1989).

[44] I. Bar-Nir et al., Nucl. Phys. B 54,17 (1973).

[45] A. Abdivaliev et al., Sov. J. Nucl. Phys. 29, 796 (1979).

[46] T. Kamae et al., Phys. Rev. Lett. 38, 468 and 471 (1977); Nucl. Phys. B 139, 394 (1978).

[47] H. Ikeda et al., Phys. Rev. Lett. 42, 1321 (1979); Nucl. Phys. B 172, 509 (1980).

[48] R. Gilman and F. Gross, arxiv:nucl-th/0111015.

[49] A. Faessler, A. J. Buchmann and M. I. Krivoruchenko, Phys. Rev. C 57, 1458 (1998).

[50] M. I. Krivoruchenko et al., Phys. At. Nucl. 74. 371 (2011) and references therein.

[51] A. W. Thomas et al., EPJ Web Conf. 63, 03004 (2013).

[52] P. Adlarson et al., Phys. Rev. C 91, 015201 (2015).

[53] P. Adlarson et al., Phys. Rev. C 86, 032201(R) (2012).

[54] R. J. Porter et al., Phys. Rev. Lett. 79, 1229 (1997).

[55] G. Agakichiev et al., Phys. Lett. B 690, 118 (2010).

[56] M. Bashkanov and H. Clement, Eur. Phys. J. 50, 107 (2014).

[57] H. Huang, J. Ping and F. Wang, Phys. Rev. C 89, 034001 (2014) and references therein.

[58] Y. Dong, P. Shen, F. Huang and Z. Zhang, arxiv:1503.02456 [nucl-th] and references therein.

[59] Hua-Xing Chen et al., arxiv:1410.0394[hep-ph].

[60] A. Gal and H. Garcilazo, Phys. Rev. Lett. 111, 172301 (2013); Nucl. Phys. A 928, 73 (2014).

[61] M. Bashkanov, Stanley J. Brodsky and H. Clement, Phys. Lett. B727, 438 (2013). 